\title{
Archéopages
}

Archéopages

Archéologie et société

Hors-série 1 | 2008

Construction ${ }^{\mathrm{s}}$ de l'archéologie

\section{Archéologie funéraire de l'âge du Fer en Champagne-Ardenne}

\section{Lola Bonnabel}

\section{(2) OpenEdition}

1 Journals

Édition électronique

URL : https://journals.openedition.org/archeopages/833

DOI : 10.4000/archeopages.833

ISSN : 2269-9872

Éditeur

INRAP - Institut national de recherches archéologiques préventives

Édition imprimée

Date de publication : 1 février 2008

Pagination : 10-15

ISSN : $1622-8545$

\section{Référence électronique}

Lola Bonnabel, « Archéologie funéraire de l'âge du Fer en Champagne-Ardenne », Archéopages [En ligne], Hors-série 1 | 2008, mis en ligne le 01 février 2008, consulté le 23 février 2023. URL : http:// journals.openedition.org/archeopages/833 ; DOI : https://doi.org/10.4000/archeopages.833 


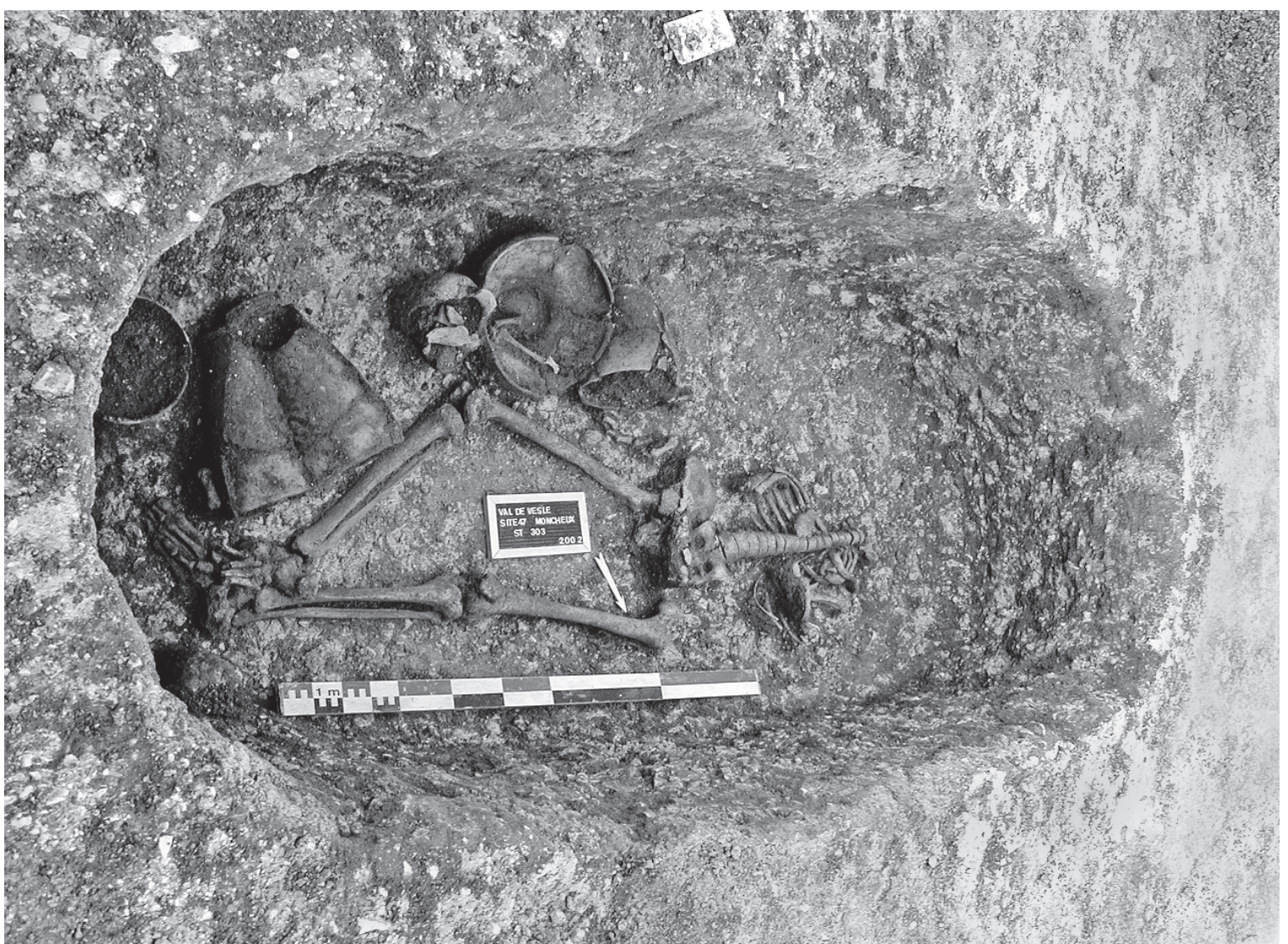

[Fig.1] Inhumation masculine

à Val de Vesle "Moncheux» (Marne)

Cette sépulture a été anciennement

sondée selon un procédé

caractéristique, appelé la sonde

champenoise: après avoir délimité

les contours de la fosse, un des angles

est de la tombe est vidé, le plus

souvent la céramique et l'essentiel

du squelette sont ainsi préservés. 
réciproque des innovations conceptuelles, méthodologiques et techniques.

Aujoulat N. 2004: Lascaux. Le geste, l'espace et le temps, Paris, Le Seuil. BINFORD L. 1983: In Pursuit of the Past, Decoding the Archaeological Record, New York, Thames \& Hudson.

BoËDA E. 2005: «Paléo-technologie ou anthropologie des Techniques?», Arob@se, www.arobase.to, vol.1, p.46-64.

BourguignON L., LHOMME V. 2004: «Les temps des chasseurscueilleurs. Le Paléolithique et le Mésolithique», in J.-P.Demoule, La France archéologique. Vingt ans d'aménagements et de découvertes, Paris, Hazan, p.36-47.

Clottes J. 2003: «De <l'art pour l'art' au chamanisme: l'interprétation de l'art préhistorique ", La Revue pour l'histoire du CNRS, nº 8 , mai 2003.

Demoule J.-P. 2004: «André Leroi-Gourhan, l'ethnie, la culture et le préhistorien: histoire d'un rendez-vous manqué», in F.Audouze, N.Schlanger (dir.), Autour de l'Homme. Contexte et actualité de Leroi-Gourhan, Antibes, Éditions APDCA, p. 45-67.

D'errico F., HenshilwoodC., Lawson G., VanhaerenM., TillieA.-M., Soressim., BressonF., MaureilleB., Nowella., LAKARraJ., BACKWELLL., JulienM. 2003: «Archaeological Evidence for the Emergence of Language, Symbolism and Music: an Alternative Multidisciplinary Perspective», Journal of World Prehistory, p.1-7o.

SAUVET G. 2004: «Langage préhistorique, langages de préhistoriens", in F.Audouze, N.Schlanger (dir.), Autour de l'Homme. Contexte et actualité de Leroi-Gourhan, Antibes, Éditions APDCA, p.249-270.

\section{Archéologie funéraire de l'âge du Fer en Champagne-Ardenne}

Lola Bonnabe

Inrap, UMR 7041 "Archéologies et Sciences de l'Antiquité»

D ans sa préface à Chronologie et société dans les nécropoles celtiques de la culture Aisne-Marne de Jean-Paul Demoule (1999), Christian Goudineau écrit: «rares furent les naufrages aussi complets que celui qui a englouti ces dizaines de milliers de tombes champenoises et l'essentiel de leur contenu: ont sombré corps et biens entre quatre et cinq cents nécropoles». La région de ChampagneArdenne a en effet été fortement occupée pendant le deuxième âge du Fer et est probablement la plus riche en sépultures de La Tène. Reste qu'il est peu d'autres régions où le passé a été aussi sauvagement ravagé.

Les travaux qui ont précédé la publication évoquée ci-dessus sont fondateurs et servent aujourd'hui de socle aux recherches sur les nécropoles de lâge du Fer en Champagne et dans la vallée de l'Aisne. Une typo-chronologie des objets a été mise en place, une culture matérielle, l'«Aisne-Marne», a été définie et une caractérisation des pratiques funéraires et de leurs variations chronologiques et régionales a été proposée. L'organisation de la société, en particulier pour ce qui est de la hiérarchie, et la relation homme-femme ont également été abordées.

Depuis les années 1990, en ChampagneArdenne, une équipe d'archéologues du funéraire gère, dans le cadre préventif, la quasi-totalité des fouilles sépulcrales, toutes périodes confondues, selon une procédure dite «protocole champenois». Parallèlement, une équipe pluridisciplinaire s'est constituée pour étudier les vestiges funéraires de l'âge du Fer. ${ }^{\mathbf{1}}$ Aujourd'hui, la diversité des types d'analyse s'accompagne d'une multiplicité de points de vue sur les vestiges, tandis que les problématiques sociétales sont de plus en plus variées.

Ce texte ne vise pas à retracer en détail l'histoire de l'archéologie funéraire de lâge du Fer en Champagne-Ardenne, mais plutôt à en évoquer les phases principales.

\section{Le $\mathrm{XIX}^{\mathrm{e}}$ siècle, l'eldorado champenois.}

Ce pourtour oriental du Bassin parisien est un gisement exceptionnel en sépultures de l'âge du Fer. Dès la fin du XviII ${ }^{e}$ siècle et surtout pendant la seconde moitié du XIX', grâce à l'impulsion donnée par Napoléon III, les sépultures sont exploitées comme de véritables mines d'objets qui, outre leur beauté intrinsèque, permettent d'alimenter une construction identitaire nationale gauloise en opposition à celle, germanique, des royaumes allemands qui, unis derrière le royaume de Prusse, ont vaincu la France en 1870. Depuis lors, la confusion entre Gaule et France n'aura cessé de nourrir le mythe d'une France millénaire, peu soucieuse de la réalité historique.

Tandis que des scientifiques notables s'intéressent à l'Antiquité classique, les acteurs des pillages ${ }^{2}$ des tombes champenoises - agissant pour le compte de plus ou moins riches commanditaires ou de leurs intermédiaires développent des techniques pour mettre en évidence les fosses sépulcrales. Leur tâche est considérablement facilitée par le contexte sédimentaire régional: les tombes sont des fosses creusées dans de la craie à la fois poreuse et dure et remplies d'une terre brune et meuble. Il suffit aux chercheurs de trésors d'observer la végétation, plus sombre et plus fournie à l'emplacement des tombes, voire d'utiliser la résonance des sabots d'un cheval. ${ }^{3}$ La plus célèbre de ces techniques - la sonde champenoise - est sans doute à l'origine des ravages les plus systématiques survenus au cours de la seconde moitié du XIX ${ }^{\mathrm{e}}$ siècle. ${ }^{4}$ Cet instrument permet de délimiter les contours des fosses sépulcrales par enfoncements successifs dans la terre végétale. Certains vivent de la vente des objets, d'autres constituent des collections ${ }^{5}$ parfois accompagnées de planches illustratives somptueuses, dont l'objectif est davantage la présentation d'objets prestigieux qu'un véritable inventaire ou qu'un classement des découvertes. Ailleurs, pourtant, les objets archéologiques sont intégrés dans des classifications construites sur les modèles naturalistes élaborés dès le XVIII ${ }^{e}$ siècle et durant le XIX ${ }^{e}$. Entre la fin du XIX ${ }^{e}$ et le début du XX $x^{e}$ siècle, les squelettes exhumés alimentent, quant à eux, les recherches des laboratoires parisiens d'anthropologie physique.

Les inventaires estimatifs des tombes sont impressionnants: trois mille cinq cents tombes en 1876, d'après Alexandre Bertrand (1820-1902), huit mille en 1881, d'après Auguste Nicaise (1828-1900) (Demoule 1999, p.15). 


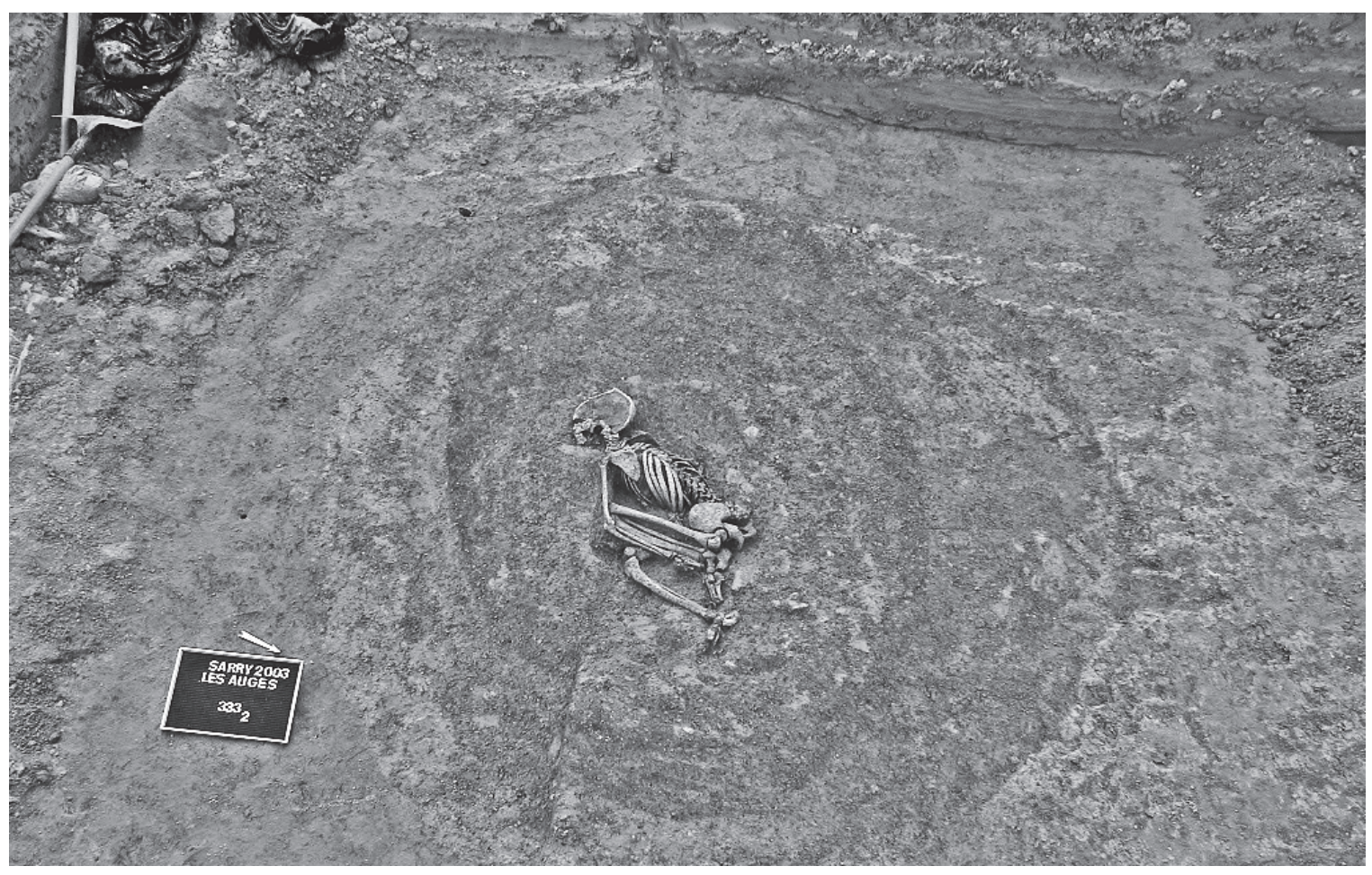

$\cong$

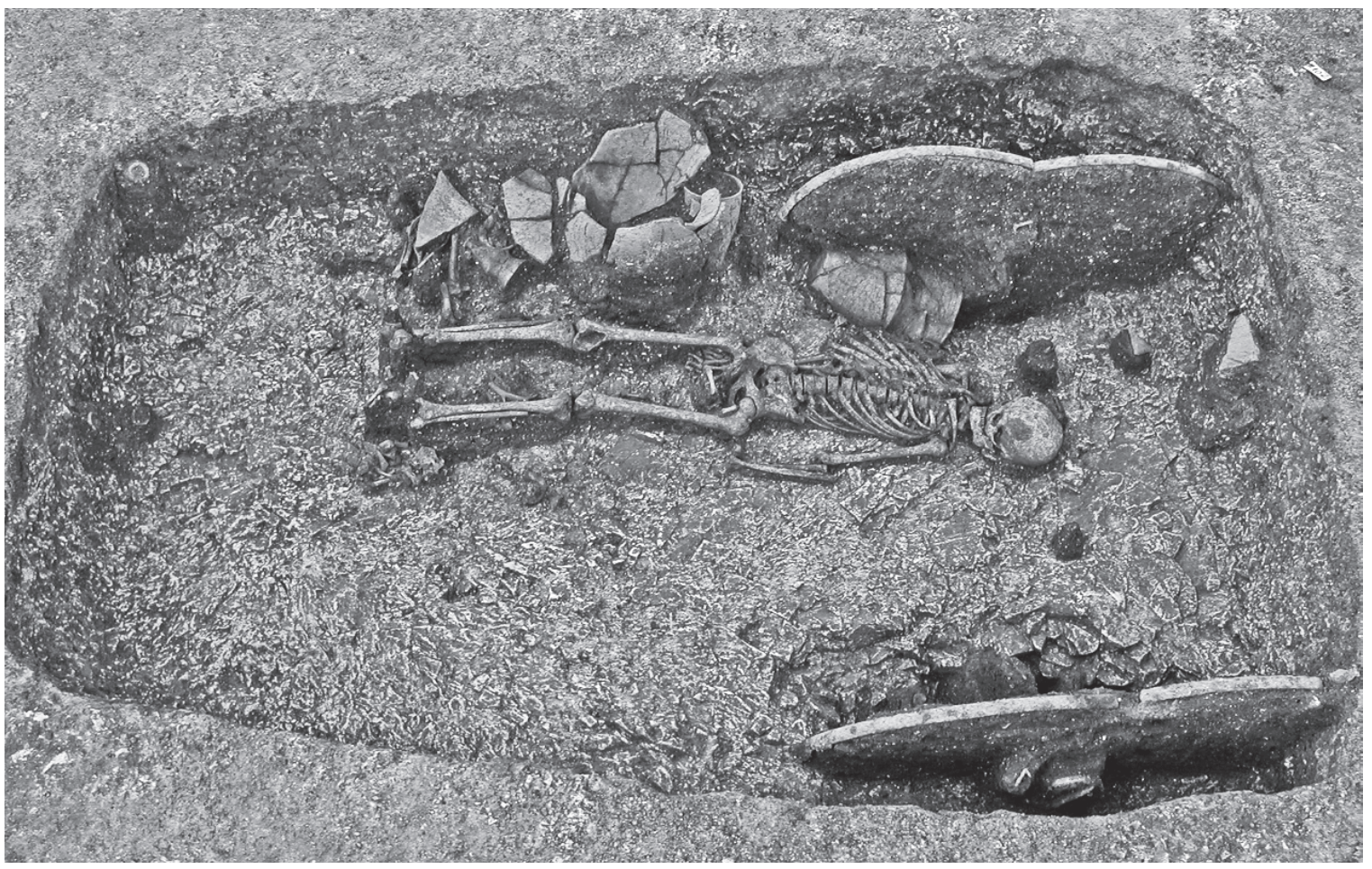

[Fig.2] Inhumation dans un silo à Sarry «Les Auges» (Marne). Les inhumations en structures réutilisées ou détournées ne peuvent plus être considérées comme anecdotiques. Aujourd'hui, hypothèse sociale et religieuse s'affrontent pour interpréter ce type de dépôts.
[Fig.3] Sépulture à char à Caurel

«Le Puisard» (Marne). Depuis 1999 sept sépultures à char ont été

fouillées et cinq autres, reconnues

en sondages, sont en attente d'être

fouillées. La fouille et l'observation

selon les procédés actuels permettent

d'identifier les mises en scène

sépulcrales ainsi que les modes de construction des chars. 
Le $\mathrm{Xx}^{\mathrm{e}}$ siècle d'une archéologie à l'autre.

Au début du $\mathrm{xx}^{\mathrm{e}}$ siècle, le développement des motivations scientifiques et patrimoniales a modifié le statut des objets. Joseph Déchelette (1908-1914) réalise un inventaire détaillé par tombe, présenté dans l'appendice de son manuel.

La notion de contexte archéologique est mise en place (Vanmoerkerke 2005, p. 28) par Léon Bérard (1883-1918). Il conserve l'ensemble des vestiges issus de chaque tombe et publie de véritables inventaires des nécropoles. Avec l'abbé PierreMarcel Favret (1875-1950), il dresse une typologie de la céramique marnienne. Comme Déchelette, il sera victime de la Première Guerre mondiale.

À partir de 1923, l'abbé Favret fouillera deux cents tombes intactes du Hallstatt final de la nécropole des Jogasses, ${ }^{\mathbf{6}}$ dont il donne la datation, même si celle-ci est encore incertaine. Les ossements humains sont devenus objets d'étude des populations. Très influencé, comme tous à l'époque, par les catégorisations raciales, il pense reconnaître sur les squelettes des Jogasses le nez typiquement hallstattien des figurations de crâne d'Europe centrale.

Sous l'impulsion de Jean Fromols (1891-1967), personnage sulfureux d'une très grande érudition archéologique, les premières fouilles d'ensembles funéraires sont conduites par André Brisson ${ }^{7}$ (1902-1970) dès les années 1930; elles ne se limitent plus aux tombes, mais englobent les enclos et les bâtiments environnants. Il ne s'agit pas seulement de dégager la fosse sépulcrale (ce qui est déjà un progrès par rapport au trou en entonnoir creusé à la tête du défunt), mais aussi d'observer l'organisation du site et d'établir des fiches d'enregistrement du squelette. ${ }^{\mathbf{8}}$ Jean Fromols

1 Les travaux de ces deux équipes sont coordonnés par l'auteur. 2 On peut considérer qu'il y a fouille et non pillage lorsqu'il y a enregistrement, dans les limites des critères scientifiques de l'époque. Cette procédure ne concerne qu'une infime minorité de tombes. 3 Demoule 1999, p.12, à propos de Léon

Foudrignier (1842-1907), inventeur de la tombe à char de Gorge Meillet. 4 Elle aurait été inventée par Jean-Baptiste Counhaye (1820-19oo) Un modèle tardif plus

sophistiqué permet

de prélever un

échantillon de sédiment;

ce procédé a été utilisé jusque dans les années 1970 pour détecter notamment

les fosses néolithiques

(Vanmoerkerke 2005,

p. 25).

5 Bien que beaucoup

plus rares, la vente

et la collection sont

toujours pratiquées

dans la région. Il est

aujourd'hui nécessaire

de recourir au

gardiennage des sites

qui, sinon, sont pillés;

tandis que quelques

est très influencé par l'archéologie allemande

(Vanmoerkerke 2007, p.34), elle-même

sous l'influence de Gustav Kossinna (1858-1931), inspirateur de l'idéologie nazie (Demoule 2002, p.17), qui fait coïncider chaque «culture» archéologique avec un groupe de population, voire une ethnie particulière. Cette superposition simpliste est encore vivace aujourd'hui.

Après la guerre, des médecins et des dentistes entreprennent des fouilles et des études anthropologiques dont il reste peu d'éléments. ${ }^{9}$

Dans les années 1960, le ministère d'André Malraux décide de faire respecter la loi de 1941 (donc promulguée sous l'occupation allemande) personnes montrent encore à leurs visiteurs des collections d'objets exposés de manière plus discrète qu'autrefois. $6 \mathrm{La}$ nécropole était connue depuis 1850

(Vatan 2004, p. 104)

7 Paysan, titulaire du certificat d'études,

il consacra toute

son énergie et sa passion

à l'archéologie. Il peut

être considéré comme

le premier archéologue

professionnel

de la région.

8 Jan Vanmoerkerke,

communication

personnelle.
9 Jan Vanmoerkerke,

communication

personnelle.

10 En particulier

Jean-Georges Rozoy,

archéologue à l'esprit

novateur et le premier,

dans la région,

à appliquer des méthodes

d'analyses systématiques

du mobilier à l'aide

de fiches perforées.

11 Comme les opérations

dirigées par P. Depaepe,

J.-L. Locht et E. Teheux

sur l'autoroute A5.

12 En particulier

d'Alain Villes

et du regretté

Erick Tappret. sur les fouilles archéologiques. Certains fouilleurs basculent alors dans la clandestinité, car l'engouement des Champenois ne sera pas stoppé par la loi. Il semble cependant qu'à partir de l'installation des services des Antiquités, en 1964, ces activités se soient considérablement ralenties. Cependant, dès les années 1970, les fouilleurs clandestins vont pouvoir bénéficier d'un nouvel instrument redoutablement efficace en matière de découverte d'objet: le détecteur de métaux. Dans le même temps, des fouilles archéologiques sur des nécropoles de La Tène sont menées par des bénévoles, ${ }^{\mathbf{1 0}}$ ainsi que par des fonctionnaires de la direction des Antiquités.

Mais, bientôt, les sites funéraires inspirent de nouvelles réflexions méthodologiques. Avec André Leroi-Gourhan puis Claude Masset et Jean Leclerc, le squelette humain devient un objet archéologique comme un autre qui permet d'identifier les gestes funéraires et les pratiques mortuaires anciennes. Sous l'impulsion d'Henri Duday, une école originale d'archéologie funéraire va alors se développer.

Mise en place de l'archéologie préventive. À la fin des années 1980, l'archéologie préventive française est en plein essor et en pleine définition. La quantité de données disponibles explose et leur exploitation, conditionnée par de puissantes contraintes de temps, est un défi scientifique. Certains chercheurs n'hésitent pas à recourir à des méthodes «révolutionnaires», comme la fouille de sites paléolithiques à la pelle mécanique. ${ }^{\mathbf{1 1}}$

Dans la région, l'essor de l'archéologie de sauvetage atteint d'abord les carrières d'exploitation de granulats, puis les chantiers des autoroutes A26 et A5. Des équipes d'archéologues professionnels sont alors créées à l'initiative des agents de l'État, ${ }^{\mathbf{1 2}}$ qui, en l'absence d'enseignement archéologique universitaire dans la région, choisissent et forment des contractuels de l'Afan. Se met alors en place une équipe constituée de personnes enthousiastes et non diplômées, dont certaines dignes héritières d'André Brisson.

Pour l'archéologie funéraire, j'ai proposé, en 1991, une nouvelle pratique de terrain sur l'A5 - qui deviendra «le protocole champenois». Il s'agit d'une technique de prélèvement quotidien rapide d'un nombre important de squelettes, permettant l'enregistrement et l'analyse des données sur place (les fiches généralement utilisées étant orientées vers un enregistrement exclusif d'analyse a posteriori). La nouvelle fiche, plutôt que d'enregistrer les ossements un à un, propose de considérer le corps par segments anatomiques; les éléments utiles à l'interprétation du mode de dépôt et des contenants funéraires sont ainsi identifiés dès le terrain. À cette fiche correspondent des modes de fouille, d'enregistrement photographique, de démontage et de mise au net des relevés constamment améliorés. $^{\mathbf{1 3}}$ 


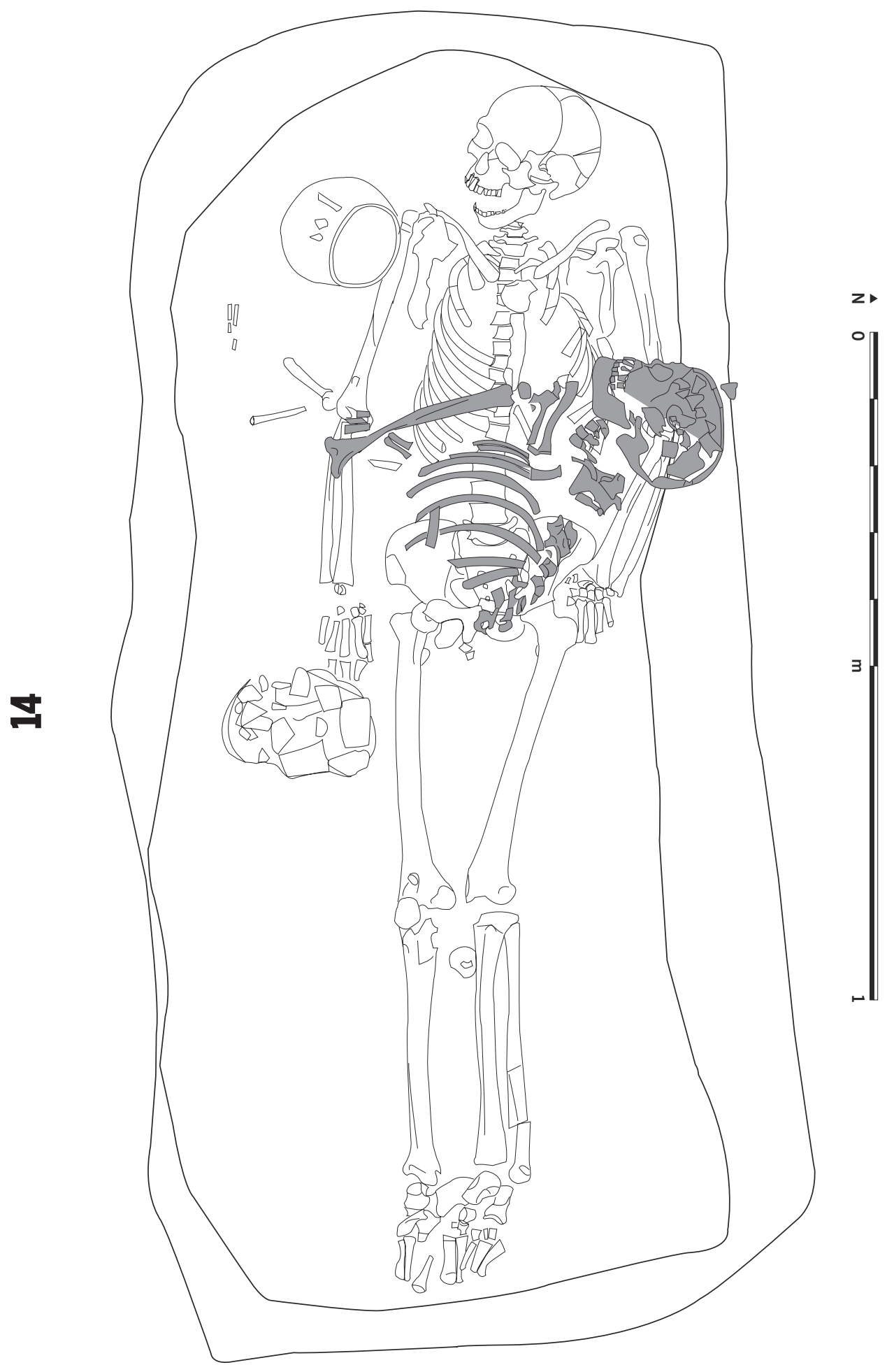

[Fig.4] Inhumations successives à

Brienne-sur-Aisne «La Croisette»

(Ardennes). La proportion

de sépultures superposées

de La Tène est très importante,

cela doit encore diminuer le nombre

d'ensemble clos anciens véritables.

À Brienne-sur-Aisne, seize

des vingt-neuf défunts sont associés

au sein de sépultures successives

ou simultanées. 

d'étude en laboratoire est élaboré l'année suivante à partir des procédures de l'anthropologie biologique (différenciations entre sexes et âges, définition de l'état sanitaire et analyse des caractères discrets), cela, afin de traiter un grand nombre de sépultures à l'aide d'observations simples et rapidement exploitables. Les résultats obtenus doivent documenter de manière pertinente les pratiques funéraires et les sociétés. ${ }^{\mathbf{1 4}}$

Ce double protocole champenois, «tanatho-archéologie et anthropologie biologique», est appliqué systématiquement depuis 1992 par une équipe constituée de chercheurs aux parcours variés. On y trouve des universitaires (archéologues, anthropologues sociaux et biologiques), et des archéologues formés «sur le tas». Cette approche systématique pratiquée par une même équipe organisée de manière cohérente a permis de répondre aux contraintes de calendrier et aux exigences scientifiques, tout en permettant des études transversales entre sites et périodes.

Les bases de données aujourd'hui disponibles - tant sur les pratiques funéraires que sur l'anthropologie biologique - concernent plus de deux mille défunts du Néolithique ancien au XIX ${ }^{\mathrm{e}}$ siècle.

\section{Vers un premier bilan pour l'âge du Fer.}

Le protocole champenois est appliqué depuis 1997 aux nécropoles de l'âge du Fer et aux défunts déposés dans des structures d'habitat. ${ }^{15}$ D'un point de vue quantitatif, le nombre de tombes fouillées n'est certes pas comparable aux dizaines de milliers sondées anciennement, dont seules deux mille sont inventoriées, mille illustrées (Demoule 1999, p.15) et à peine trois cents scientifiquement exploitables aujourd'hui. Une grande partie des sépultures fouillées dans le cadre de l'archéologie préventive ont été anciennement perturbées. Néanmoins, et c'est peut-être le point essentiel, toutes ces structures ont livré des informations qui participent au renouvellement de l'image que nous avons des pratiques funéraires de l'âge du Fer. De plus, la multiplicité des types d'analyse

13 Les progrès

de l'iconographie, tant pour

l'enregistrement

que pour la présentation

des données, sont

dus à Sylvie Culot

et Babeth Vauquelin.

14. Ce protocole

a été élaboré en 1993

dans le cadre

d'un DEA (université

Bordeaux I)

Anthropologie biologique

et sauvetage. Une étude

de cas: le cimetière

médiéval d'Isle-sur-Suippe

(51); il s'est beaucoup

inspiré du travail

d'Hervé Guy, Inrap.

fouilles programmées

menées par B.Lambot

sont d'un apport

fondamental.

16 Par ordre

Achard-Corompt,

Ginette Auxiette,

Lola Bonnabel, Sylvie

Culot, Sophie Desenne,

Vincent Garénaux,

Christelle Lagatie,

Catherine Moreau,

Cécile Paresys, Isabelle

Richard, Marion Saurel,

Élisabeth Vauquelin.
Pour compléter ce dispositif, un protocole

opérés grâce aux progrès méthodologiques de l'archéologie augmente considérablement le nombre de données sur lesquelles appuyer nos analyses et nos hypothèses.

Ces recherches s'inscrivent dans les problématiques générales de l'archéologie: les sites sont décapés sur de grandes surfaces, ils se situent dans un paysage où sondages et décapages ont ouvert des fenêtres sur des milliers d'hectares. Il faut souligner à cet égard que les sondages, qualifiés à tort de négatifs, participent pleinement à l'étude des territoires anciens.

Le corpus élaboré au cours des dix dernières années est constitué d'environ cinq cents défunts inhumés (moins d'une dizaine ont été préalablement incinérés), au sein de sépultures parfois isolées mais le plus souvent intégrées dans un groupe de tombes numériquement variables (de trois à quatre-vingt-trois sépultures). À ces défunts inhumés (selon des normes «majoritaires») s'ajoutent des défunts traités de manière particulière mais non anecdotique, puisque les restes osseux d'une quarantaine d'entre eux ont été retrouvés au sein de structures d'habitat réutilisées, voire détournées.

Un projet de recherche réunit actuellement les chercheurs qui ont étudié ces ensembles. ${ }^{\mathbf{1 6}}$ La synthèse des données - thanato-archéologiques, objets céramiques et métalliques, archéozoologie, anthropologie biologique, topographique et organisation fonctionnelle des sites et des occupations à l'échelle régionale - permettra de proposer une description précise des pratiques funéraires et de leurs variations en fonction des critères sociaux, géographiques et chronologiques. Les plus anciens de ces dépôts funéraires ou apparentés appartiennent à l'extrême fin de l'âge du Bronze final et au début de l'âge du Fer. La plupart d'entre eux sont à rattacher à une chronologie qui s'étend du $\mathrm{V}^{\mathrm{e}}$ au $\mathrm{III}^{\mathrm{e}}$ siècle avant notre ère. La quarantaine de sites concernés se trouvent majoritairement dans la plaine crayeuse, surtout dans la Marne ainsi que dans l'Aube et les Ardennes.

DÉCHELETTE J. 1908-1914: Manuel d'archéologie préhistorique, celtique et gallo-romaine, IV, Paris, A.Picard et fils, 2 tomes en $6 \mathrm{vol}$., dont 2 vol. d'appendices (plusieurs rééditions).

Demoule J.-P. 1999: "Chronologie et Société dans les nécropoles celtiques de la culture Aisne-Marne, du VI ${ }^{e}$ au III ${ }^{e}$ siècle avant notre ère», Revue Archéologique de Picardie, numéro spécial, 15.

Demoule J.-P. 2002: «La responsabilité des archéologues dans la construction des nationalismes modernes», Raison présente, 142 , p.15-30.

à ces recherches, diverse

alphabétique: Nathalie

VANMOERKERKE J. 2005: «Historique des recherches autour de Vatry», in Europort Vatry (Marne) Les pistes de l'archéologie, Quand la plaine n'était pas déserte, Langres, Éditions Dominique Guéniot, 2005, p. 25-33.

VANMOERKERKE J. 2007: «Jean Fromols et l'histoire de l'archéologie champenoise pendant la Seconde Guerre Mondiale», in J.-P.Legendre, L.Olivier, B.Schnitzler (dir.), L'Archéologie nazie en Europe de l'Ouest, Actes de la table ronde internationale «Blut und Boden» tenue à Lyon dans le cadre du X ${ }^{e}$ congrès de l'EAA le 8 et 9 septembre 2004, p.163-176.

VATAN A. 2004: Histoire de larchéologie celtique en Champagne. Des origines à nos jours, Mémoire de la Société archéologique champenoise, $\mathrm{n}^{\circ} 17$. 